\title{
Expression and subcellular localization of menin in human cancer cells
}

\author{
FENG REN $^{1}$, HONG-WEI XU ${ }^{2}$, YU HU ${ }^{1}$, SHUANG-HONG YAN ${ }^{1}$, \\ FENG WANG ${ }^{1}$, BAO-WEI SU ${ }^{2}$ and $\mathrm{QI} \mathrm{ZHAO}^{2}$ \\ Departments of ${ }^{1}$ Clinical Laboratory, and ${ }^{2}$ General Surgery, \\ The Fourth People's Hospital of Wuxi, Wuxi, Jiangsu 214062, P.R. China
}

Received January 19, 2012; Accepted March 21, 2012

DOI: $10.3892 / \mathrm{etm} .2012 .530$

\begin{abstract}
The aim of this study was to elucidate the expression and localization of menin, a protein encoded by the multiple endocrine neoplasia type I (MEN1) gene, in 13 human cancer cell lines. Reverse transcription-polymerase chain reaction (RT-PCR) was used to determine the expression of the menin gene. The localization of the menin protein was detected by immunofluorescence microscopy. Western blotting was used to determine the quantity of menin in the nucleus, cytosol and membrane of the cells. RT-PCR revealed that menin was expressed in all the cell lines examined in this study. Immunofluorescence microscopy revealed that menin was located primarily in the nucleus. In the GES-1 (transformed human gastric epithelium), MCF-7 (breast cancer), SGH44 (brain glioma) and HeLa (cervical cancer) cell lines, menin was also found to be localized to the membrane, cytosol and nucleus. Moreover, in SGH44 cells more menin was located in the cytosol than the nucleus. Similar findings were obtained by western blotting. In the GES-1 and MKN-28 cells undergoing octreotide treatment, cytoplasmic menin was significantly increased compared with the control groups. Therefore, we suggest that menin is expressed in a number of human cancer cell lines and that the cytosolic distribution increases when the cells undergo octreotide treatment, indicating a new role for menin.
\end{abstract}

\section{Introduction}

Menin, encoded by the multiple endocrine neoplasia type I (MEN1) gene, is a nuclear protein which is mutated in patients with a dominantly inherited disorder characterized by the appearance of combinations of tumors in a number of endocrine tissues, including the pituitary and parathyroid glands and the pancreas $(1,2)$, and occasionally in non-endocrine

Correspondence to: Mr. Feng Ren, Department of Clinical Laboratory, The Fourth People's Hospital of Wuxi, 200 Huihe Road, Wuxi, Jiangsu 214062, P.R. China

E-mail: renfeng198254@163.com

Key words: menin, cancer cells, subcellular localization, nucleus glands $(3,4)$. The human MEN1 gene has been identified by positional cloning and is localized to chromosome 11q13. The gene consists of 10 exons and codes for an mRNA $2.8 \mathrm{~kb}$ in length (5). The protein product of the MEN1 gene, menin, is highly conserved in organisms from Drosophila to humans. As menin does not show a clear homology to any known protein motifs, it has been challenging to elucidate the mechanism by which menin acts as a tumor suppressor (6).

Numerous studies have revealed that menin is mainly located in the nucleus and possesses nuclear localization signals (NLSs) in its carboxy-terminal region (7). It has also been reported that menin is found in membrane and cytoplasmic fractions (8), although at lower levels than in nuclear fractions, indicating that menin may also play a role outside of the nucleus. Multiple lines of evidence suggest that menin, as a scaffold protein, interacts with cytoskeletal proteins, including glial fibrillary acid protein (GFAP), vimentin (8) and IQGAP1 (9). However, less is known concerning the expression and biochemical function of menin expression in non-endocrine cells. To elucide the mechanism by which menin functions as a tumor suppressor, in the present study, we report the detection of endogenously expressed menin in 13 human cancer cell lines. In particular, we further investigated the subcellular localization of menin in cancer cell lines.

\section{Materials and methods}

Cell lines and cell culture. The following cell lines were obtained from the Institute of Cell Biology of the Chinese Academy of Sciences (Shanghai, China): AGS, BGC-823 and SGC-7901 (human gastric cancer), Huh7 and Hep3B2.1-7 (liver cancer), MCF-7 and MDA-MB-231 (breast cancer), SW480 (colon cancer), SGH44 (brain glioma), SKOV-3 (ovarian cancer) and HeLa (cervical cancer). The transformed human gastric epithelial cell line GES-1 was a gift from Dr Yong-Chang Chen (Jiangsu University, China). The MNK-28 cell line was a gift from Dr Xiao-Ying Li (Rui-Jin Hospital, Shanghai Jiao-Tong University School of Medicine, Shanghai, China). The cells were cultured in Dulbecco's modified Eagle's medium (DMEM; Gibco, Grand Island, NY, USA) supplemented with $10 \%$ newborn calf serum (NBCS; Minhai Bio-engineering Co., Lanzhou, China) and maintained at $37^{\circ} \mathrm{C}$ in a humidified atmosphere of $5 \% \mathrm{CO}_{2}$. The medium was 
changed every two days and the cells were subcultured until reaching confluency.

RNA extraction and reverse-transcription polymerase chain reaction $(R T-P C R)$. Total RNA was extracted using TRIzol reagents (Invitrogen, Carlsbad, CA, USA) according to the manufacturer's instructions. Total RNA $(1 \mu \mathrm{g})$ was reverse transcribed into cDNA using dNTPs $(1 \mathrm{mM}), 5 \mathrm{X}$ reverse transcription buffer (500 mM Tris-HCL pH 8.3, $250 \mathrm{mM}$ $\mathrm{KCl}, 50 \mathrm{mM} \mathrm{MgCl}_{2}$ and $50 \mathrm{mM}$ DTT), 16 units RNasin and 2.5 units of AMV reverse transcriptase (Gibco BRL, Life Technologies, Carlsbad, CA, USA). PCR was carried out in a total volume of $20 \mu \mathrm{l}$, containing $2 \mu \mathrm{l} 10 \mathrm{X}$ buffer, $0.4 \mu \mathrm{ldNTP}$, $0.5 \mu \mathrm{l}$ primers, $1 \mu \mathrm{l}$ cDNA, $0.2 \mu \mathrm{l}$ Taq enzyme and $15.4 \mu \mathrm{l} \mathrm{H}_{2} \mathrm{O}$ (Shenergy Biocolor Biological Science and Technology Co., Shanghai, China). The specific primers were: menin gene, forward 5'-GCCTGGGTAGTGTTTGGGC-3' and reverse 5'-CACAGCGCATGTATGATCCTT-3', product of $452 \mathrm{bp}$; $\beta$-actin, forward 5'-TGCGTGACATTAAGGAGAAG-3' and reverse 5'-GCTCGTAGCTCTTCTCCA-3', product of $247 \mathrm{bp}$. Following an initial denaturation step of $5 \mathrm{~min}$ at $95^{\circ} \mathrm{C}$, 30 cycles of amplification for the primer pairs were carried out. Each cycle included a denaturation step of $30 \mathrm{sec}$ at $95^{\circ} \mathrm{C}$, annealing for $1 \mathrm{~min}$ at $55^{\circ} \mathrm{C}$ and an elongation step of $1 \mathrm{~min}$ at $72^{\circ} \mathrm{C}$, with a final extension for $5 \mathrm{~min}$ at $72^{\circ} \mathrm{C}$. The products were separated on $1.5 \%$ agarose gel and the relative gene expression was measured using a digital image (Perkin-Elmer, Wellesley, MA, USA). The experiments were performed in triplicate and the mean value was calculated.

Immunofluorescence microscopy. The cells grown on coverslips were fixed with freshly prepared paraformaldehyde $(40 \mathrm{~g} / \mathrm{l}$ in PBS) for $1 \mathrm{~h}$ prior to being penetrated with $0.3 \%$ Triton $\mathrm{X}-100$ and blocked with $3 \%$ bovine serum albumin (BSA) in PBS. The cells were then incubated with the primary antibody at $4^{\circ} \mathrm{C}$ overnight and then with the $\mathrm{Cy} 3$-conjugated secondary antibody for $1 \mathrm{~h}$ at room temperature (RT), with three washes following each incubation. The distribution of the target protein in the cells was analyzed by fluorescence microscopy.

Preparation of nuclear and cytoplasmic samples. The cells were extracted by Dounce homogenization in HEM buffer (10 mmol/l HEPES pH 7.5, 2 mmol/l EDTA, 1 mmol/ $/ \mathrm{MgCl}_{2}$ ) as described previously (10). The homogenate was centrifuged at $500 \mathrm{xg}$ at $4^{\circ} \mathrm{C}$ for $5 \mathrm{~min}$ to obtain the nuclear proteins and the supernatant was centrifuged at $37,000 \mathrm{x} \mathrm{g}$ at $4^{\circ} \mathrm{C}$ for $30 \mathrm{~min}$. The supernatant and the pellet from the second centrifugation are referred as cytosol and membrane preparations, respectively. The membrane preparation was washed twice with HEM buffer to remove contaminating cytosol. The protein concentrations were determined and equal amounts of protein from each preparation (30 $\mu \mathrm{g}$ ) were subjected to SDS-PAGE.

Western blotting. Sample proteins were run on $10 \%$ SDS-polyacrylamide gels and transferred to a polyvinylidene difluoride membrane (PVDF, Amersham, Piscataway, NJ, USA) by electronic transfer. The membranes were blocked with 5\% non-fat dried milk and then incubated with an antibody against menin (dilution 1:200) and GAPDH (dilution $1: 5,000)$ overnight at $4^{\circ} \mathrm{C}$ and with the secondary antibody
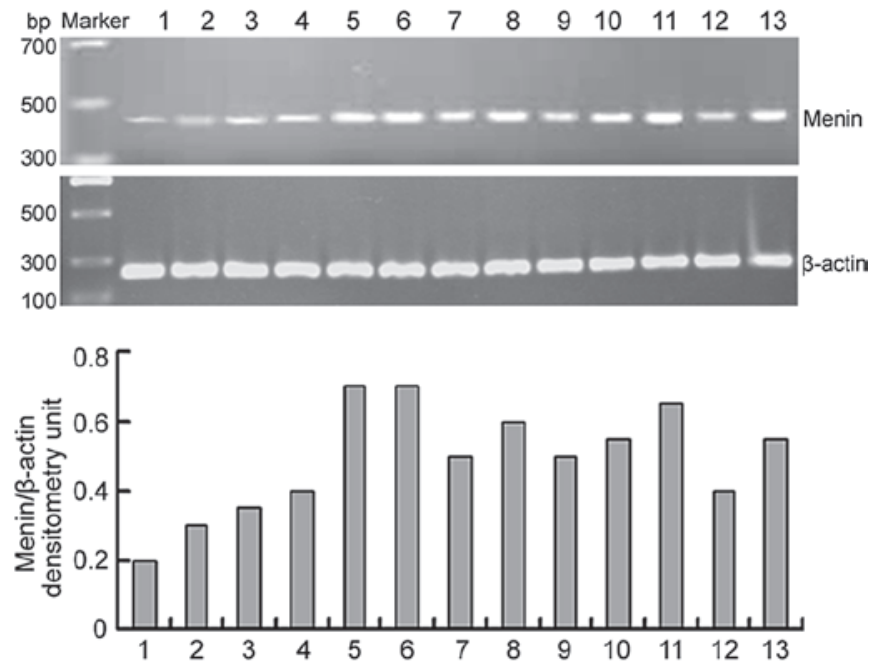

Figure 1. Expression of menin mRNA in 17 human cancer cell lines was detected by RT-PCR. The 452-bp human menin-specific sequence and a 247-bp $\beta$-actin sequence were amplified from the cDNA of cancer cell lines, separated by agarose gel electrophoresis and visualized by ethidium bromide staining. Densitometry of menin transcripts was standardized to $\beta$-actin. Lane 1, AGS; lane 2, BGC-823; lane 3, SGC-7901; lane 4, MKN-28; lane 5, GES-1; lane 6, Huh7; lane 7, Hep3B2.1-7; lane 8, MCF-7; lane 9, MDA-MB-231; lane 10, SW480; lane 11, SGH44; lane 12, SKOV-3; lane 13, HeLa. RT-PCR, reverse transcription-polymerase chain reaction.

for $1 \mathrm{~h}$ at RT, with three washes following each incubation. Electrochemiluminescence reagents were used to show the positive bands on the membrane. The bands on film were analyzed with GeneSnap/Gene Tool software from Syngene (Cambridge, UK).

Statistical analysis. All of the numerical data are expressed as the mean $\pm \mathrm{SD}$. Statistical analysis was performed using SPSS 16.0 edition program (SPSS Inc., Chicago, IL, USA) for ANOVA with the Scheffé multiple comparison test. $\mathrm{P}<0.05$ was considered to indicate a statistically significant result.

\section{Results}

Expression of menin mRNA in human cancer cells. As shown in Fig. 1, menin mRNA was examined in 13 human cancer cell lines. RT-PCR results revealed that menin was positively expressed in all the cell lines examined in this experiment. In the gastric cancer cell lines, the expression of menin mRNA was gradually increased from AGS, BGC-823, SGC-7901 and MNK-28 to GES-1. It was significantly higher in GES-1 than the other four gastric cancer cell lines $(\mathrm{P}<0.05)$. The expression of menin may be correlated with the malignancy of the cells.

Menin is generally distributed in cancer cell lines. The expression of the menin protein in cancer cell lines was further confirmed by immunoblotting, which revealed a $74-\mathrm{kDa}$ protein band. The results also revealed that the menin protein was expressed in different cancer cell lines (Figs. 2 and 3). Moreover, the menin protein was expressed not only in the nucleus, but also in the cytosol and membrane in the GES-1, MCF-7, SGH44 and HeLa cell lines (data not shown). The expression of the menin protein was significantly higher in 


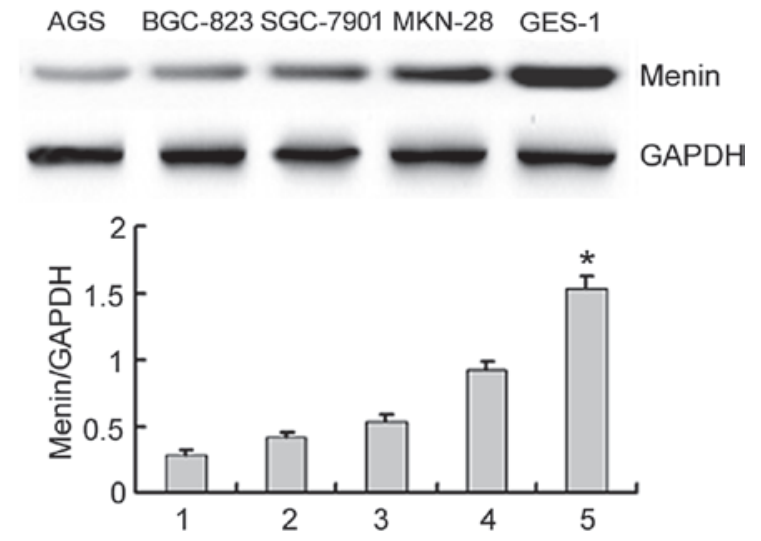

Figure 2. Expression of menin protein in gastric cancer and GES-1 cell lines. The expression of menin and GAPDH was detected by western blotting. The volume ratio of menin/GAPDH was calculated and presented. Each bar represents mean \pm SD obtained from three independent experiments. Compared with the other four gastric cancer cell lines, the expression of menin in GES-1 cells was higher ("P<0.05). Bar graph: 1, AGS, 2, BGC-823; 3, SGC-7901; 4, MKN-28; 5, GES-1.

GES-1 cells than in the other gastric cancer cell lines $(\mathrm{P}<0.05$; Fig. 2).

The subcellular localization of menin in different cancer cell lines. Immunofluorescence microscopy revealed that menin was located primarily in the nucleus. However, in the four human cancer cell lines (GES-1, MCF-7, SGH44 and HeLa), menin was localized not only in the nucleus, but also in the cytosol and membrane. Moreover, in the SGH44 cells more menin was located in the cytosol than that in the nucleus (Figs. 4 and 5).

More menin protein is distributed in the cytosol with octreotide treatment. The relative protein expression levels of menin in the GES-1 and MKN-28 cell lines were significantly increased in the octreotide group compared with the control group. Moreover, more menin protein was located in the cytosol with octreotide treatment (Fig. 6).

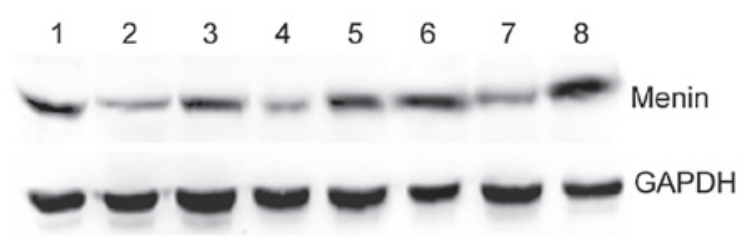

Figure 3. Expression of menin in human cancer cell lines. The cell extract was constructed and menin and GAPDH were detected by western blotting. The expression of menin in Hep3B2.1-7, MDA-MB-231 and SKOV-3 cells was decreased compared with the other cell lines. Lane 1, Huh7; lane 2, Hep3B2.1-7; lane 3, MCF-7; lane 4, MDA-MB-231; lane 5, SW480; lane 6, SGH44; lane 7, SKOV-3; lane 8, HeLa.

\section{Discussion}

The present study detected the expression and distribution of menin in 13 cancer cell lines, including AGS, BGC-823, SGC-7901 and MNK-28 (gastric cancer), GES-1 (transformed gastric epithelium), Huh7 and Hep3B2.1-7 (liver cancer), MDA-MB-231 and MCF-7 (breast cancer), SW480 (colon cancer), SKOV-3 (ovarian cancer), HeLa (cervical cancer) and SGH44 (brain glioma) cells. The results of RT-PCR, western blotting and immunofluorescence microscopy revealed that menin was positively expressed in all of the cell lines examined in this study. The nuclear localization of the menin protein was extensive and general, with a discrepancy between the gastric cancer and transformed gastric epithelial cell lines and the cancer and SGH44 (brain glioma) cell lines. Furthermore, we found that the somatostatin analog octreotide increased the expression of menin, particularly in the cytosol. Consistent with the findings of Mensah-Osman et al (11), octreotide induces menin expression by the suppression of PKA activation.

The MEN1 gene encodes the menin protein, which is thought to be involved in a number of mechanisms that are dysregulated in cancer cells, including genome stability and the regulation of gene transcription, cell proliferation and apoptosis (12-15). These diverse menin functions were largely attributed to the crucial role of menin in endocrine cells and tissues. The results of the present study support the hypothesis that the nucleus-cytoplasm-membrane treble distribution of

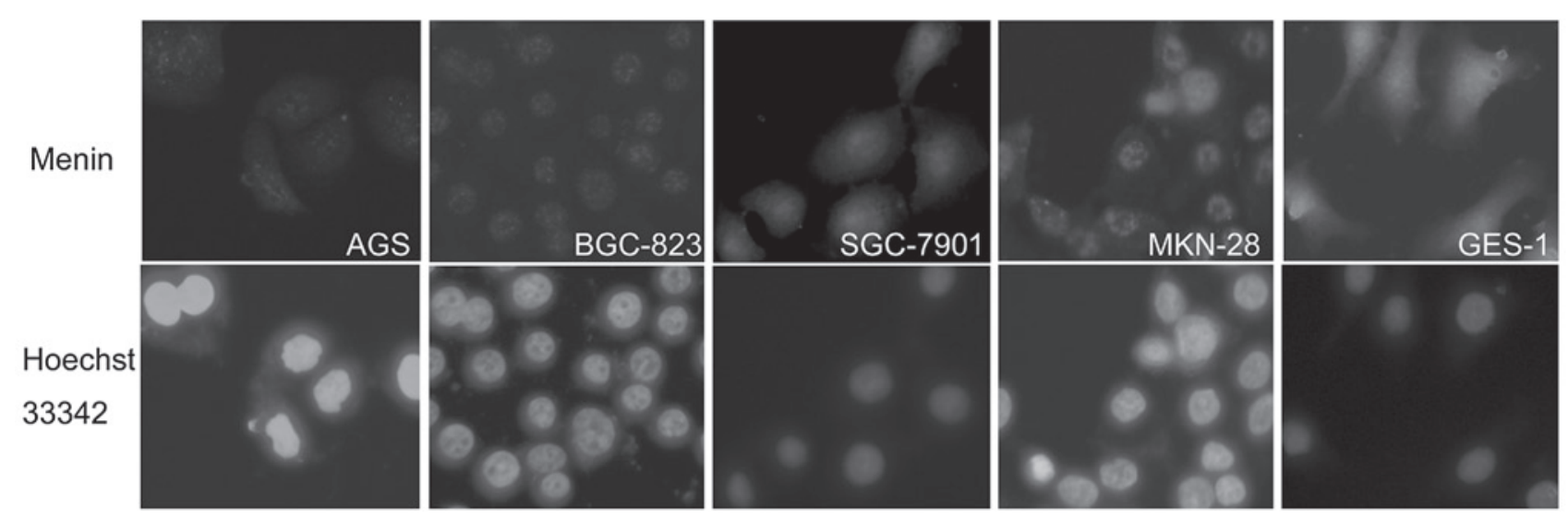

Figure 4. Nuclear localization of menin in gastric cancer and GES-1 cell lines. The cell lines were immunofluorescently stained with a specific fluorescence antibody against menin and the results showed that in all the cell lines, menin is located primarily in the nucleus. In the GES-1 cells, menin was also found to be localized to the membrane, cytosol and nucleus (original magnification, $\mathrm{x} 200$ ). 

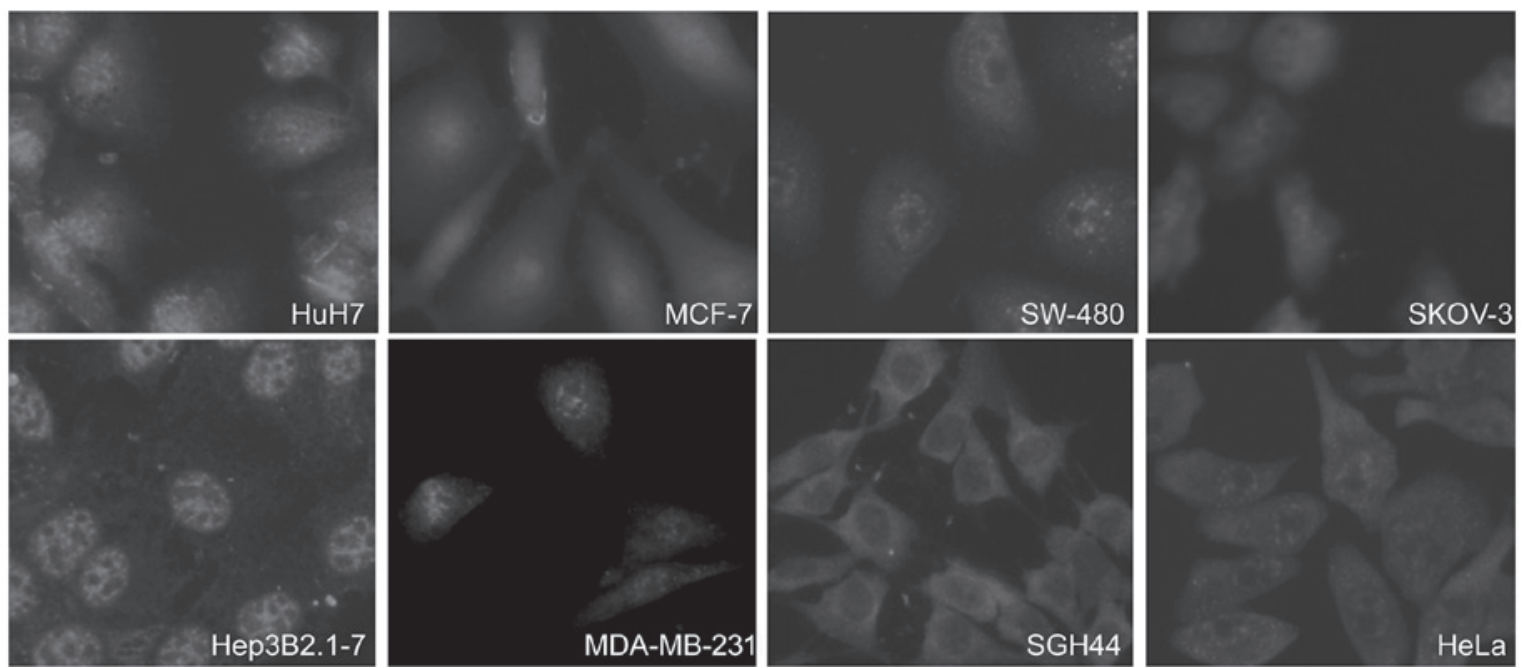

Figure 5. Localization of menin in different cancer cell lines. The cell lines were immunofluorescently stained with a specific fluorescence antibody against menin and the results showed that in all cell lines, menin was localized to the membrane, cytosol and mainly the nucleus. However, the expression of menin was higher in the cytosol than that in the nucleus in SGH44 brain glioma cells (original magnification, x200).

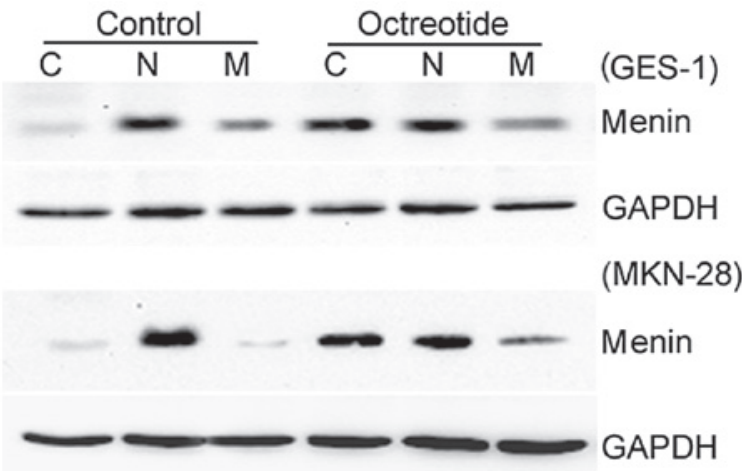

Figure 6. Expression of menin in GES-1 and MKN-28 cells treated with or without octreotide. Western blotting: GES-1 and MKN-28 cells were lysed and cytosolic $(\mathrm{C})$, nuclear $(\mathrm{N})$ and membrane $(\mathrm{M})$ fractions were obtained. The expression of menin and GAPDH in each fraction was detected Compared with the control, the expression of menin in the cytosol was significantly increased in octreatide-treated cells.

menin was a general phenomenon and that more menin protein was localized to the nucleus of non-endocrine cancer cells. Although the primary function of menin as a regulator of gene transcription, cell proliferation, apoptosis and genome stability has been determined $(16,17)$, the role of menin protein in the cytoplasm-membrane localization remains unknown. These observations provide novel insights into how menin suppresses tumorigenesis.

Menin is predominantly located in the nucleus and contains two classic NLSs, NLS1 and NLS2 (18). Classic NLSs comprise positively charged amino acid residues which bind to a soluble transport receptor complex made up of importins $\alpha$ and $\beta$, causing the protein to be translocated to the nucleus (19). The nuclear localization of menin is thought to be necessary for its ability to regulate gene transcription, as the protein regulates gene expression by associating with chromatin and the nuclear matrix and binding double-stranded DNA (20). Whereas, the simultaneous deletion of NLS1 and NLS2 in menin attenuates menin translocation into the nucleus, on the contrary, accumulating quantities of menin protein are noted in the cytosol. Our results showed that menin was also localized to the cytosol and membrane in GES-1, MCF-7, SGH44 and HeLa cells. In particular, the level of expression of menin was higher in the cytosol than in the nucleus in SGH44 cells. These results suggest that menin NLS deletion mutants and the deletion of a stretch of amino acid residues may affect the expression and general structure of menin. On the other hand, menin has several NLSs, suggesting that its transcriptional activity may be regulated by its ability to move in and out of the nucleus (18).

Mensah-Osman et al (11) found that octreotide-suppressed PKA activation may markedly increase the numbers of menin-expressing cells and levels of menin mRNA and menin protein expression. This study also revealed that when the cells were treated with octreotide, the expression of the menin protein was increased and the protein was rapidly transported out of the nucleus and congregated in the cytosol and on the membrane. Generally, stimuli that promote the expression of a protein are also able to promote its intracellular movement (most are cytoplasm-to-nucleus translocations) $(21,22)$. Octreotide may promote the reverse translocation of the menin protein from the nucleus to the cytoplasm. Consistent with our results, Yan et al (9) observed the co-localization of IQGAP1 with a non-nuclear pool of menin in $\beta$ cell lines. The co-localization of menin at the plasma membrane was observed to be extensive, particularly at cell-cell junctions. The authors also reported that menin interacts with IQGAP1, a scaffold protein, reducing its interaction with GTP-Rac1 and increasing its interaction with E-cadherin/ $\beta$-catenin. This suggests the existence of a menin-IQGAP1 pathway which influences cell migration and cell-cell adhesion in endocrine tissue. According to its new localization, our results provide new insight into some unknown functions of menin protein in non-endocrine cells.

Together, these results demonstrated that menin was positively expressed in all of the cell lines examined in this study. 
The nuclear localization of the menin protein was extensive and general and its expression in the cytoplasm may play a more significant role in coordinating the activation and repression of gene transcription than merely targeting menin to the nucleus. The precise roles of menin in the cytoplasm and the membrane are not yet fully understood. Further detailed analysis must be carried out to establish the functions of menin and its role in the cytoplasm.

\section{Acknowledgements}

This study was supported by grants from the Natural Science Foundation of Jiangsu Province (no. BK2008115) and the Medical Science Foundation of Wuxi, Jiangsu Province (no. YGM1111).

\section{References}

1. Wu T and Hua X: Menin represses tumorigenesis via repressing cell proliferation. Am J Cancer Res 1: 726-739, 2011.

2. Pannett AA and Thakker RV: Multiple endocrine neoplasia type 1. Endocr Relat Cancer 6: 449-473, 1999.

3. Tsukada T, Nagamura Y and Ohkura N: MEN1 gene and its mutations: basic and clinical implications. Cancer Sci 100 209-215, 2009.

4. Feng ZJ, Gao SB, Wu Y, Xu XF, Hua X and Jin GH: Lung cancer cell migration is regulated via repressing growth factor PTN/ RPTP $\beta / \zeta$ signaling by menin. Oncogene 29: 5416-5426, 2010.

5. Hory B and Drüeke TB: Menin and MEN 1 gene: a model of tumour suppressor system. Nephrol Dial Transplant 13 2176-2179, 1998.

6. Yang Y and Hua X: In search of tumor suppressing functions of menin. Mol Cell Endocrinol 265-266: 34-41, 2007.

7. Guru SC, Goldsmith PK, Burns AL, et al: Menin, the product of the MEN1 gene, is a nuclear protein. Proc Natl Acad Sci USA 95: 1630-1634, 1998.

8. Lopez-Egido J, Cunningham J, Berg M, Oberg K, BongcamRudloff E and Gobl A: Menin's interaction with glial fibrillary acidic protein and vimentin suggests a role for the intermediate filament network in regulating menin activity. Exp Cell Res 278: $175-183,2002$
9. Yan J, Yang Y, Zhang H, et al: Menin interacts with IQGAP1 to enhance intercellular adhesion of beta-cells. Oncogene 28: 973-982, 2009.

10. Chen YC, Ren F, Sang JR, Tao Y and Xu WR: Type II cGMPdependent protein kinase inhibits proliferation of the gastric cancer cell line BGC-823. Mol Med Report 3: 361-366, 2010.

11. Mensah-Osman E, Zavros Y and Merchant JL: Somatostatin stimulates menin gene expression by inhibiting protein kinase $\mathrm{A}$. Am J Physiol Gastrointest Liver Physiol 295: G843-G854, 2008.

12. Sowa H, Kaji H, Hendy GN, et al: Menin is required for bone morphogenetic protein 2- and transforming growth factor betaregulated osteoblastic differentiation through interaction with Smads and Runx2. J Biol Chem 279: 40267-40275, 2004.

13. Kim YS, Burns AL, Goldsmith PK, et al: Stable overexpression of MEN1 suppresses tumorigenicity of RAS. Oncogene 18: 5936-5942, 1999.

14. La P, Yang Y, Karnik SK, et al: Menin-mediated caspase 8 expression in suppressing multiple endocrine neoplasia type 1. J Biol Chem 282: 31332-31340, 2007.

15. Guru SC, Crabtree JS, Brown KD, et al: Isolation, genomic organization, and expression analysis of Men1, the murine homolog of the MEN1 gene. Mamm Genome 10: 592-596, 1999.

16. Zhang H, Li W, Wang Q, et al: Glucose-mediated repression of menin promotes pancreatic $\beta$-cell proliferation. Endocrinology 153: 602-611, 2012.

17. Francis J, Lin W, Rozenblatt-Rosen O and Meyerson M: The menin tumor suppressor protein is phosphorylated in response to DNA damage. PLoS One 6: e16119, 2011.

18. La P, Desmond A, Hou Z, Silva AC, Schnepp RW and Hua X: Tumor suppressor menin: the essential role of nuclear localization signal domains in coordinating gene expression. Oncogene 25: 3537-3546, 2006

19. Komeili A and O'Shea EK: New perspectives on nuclear transport. Annu Rev Genet 35: 341-364, 2001.

20. La P, Silva AC, Hou Z, et al: Direct binding of DNA by tumor suppressor menin. J Biol Chem 279: 49045-49054, 2004.

21. Tell G, Damante G, Caldwell D and Kelley MR: The intracellular localization of APE1/Ref-1: more than a passive phenomenon? Antioxid Redox Signal 7: 367-384, 2005.

22. Li Y, Chen Y, Tao Y, Xu J and Chen M: RhoA protein is generally distributed in the nuclei of cancer cells. Oncol Rep 24: 1005-1009, 2010 . 